\title{
EXAMINATION OF SMEARS FOR TUBERCLE BACILLI BY FLUORESCENCE MICROSCOPY
}

\author{
Else Holst, D. A. Mitchison, and S. Radhakrishna \\ (From the Tuberculosis Chemotherapy Centre*, Madras)
}

Reprinted, by kind permission, from Ind. Jour. Med. Res. 47, 5;

September, 1959, pp. 495-499.

In underdeveloped countries, laboratory facilities for the bacteriological diagnosis of tuberculosis are, at present, very limited. Cultural methods are unlikely to be used on a large scale for many years to come. It is, therefore, important to investigate the most economical method of examining smears for tubercle bacilli. Fluorescence microscopy was introduced by Hagemann (1937) and has since been described by many authors, including Tanner (1941, 1948), Line and Shaughnessy (1941), Lempert (1944), Norman and Jelks (1945), Clegg and Foster-Carter (1946), Wilson (1952), Von Haebler and Murray (1954), and Needham (1957). The great advantage claimed for this method is that stained bacilli can be detected using a much lower magnification than with the usual Ziehl-Neelsen method. Considerable time is saved in examining smears and larger areas can be searched. The method has not been widely employed for two reasons. In the first place, the light source must be very bright and many of the optical systems described previously have only supplied sufficient light if the equipment was used in a darkened room. Secondly, some workers (Ritterhoff and Bowman, 1945; Kuster, 1939; Holm and Plum, 1943) consider that false positive results can be obtained, since some smears may contain small naturally fluorescent particles which can be confused with bacilli.

Equipment for fluorescence microscopy that can be used in normal daylight has been in use at the Tuberculosis Chemotherapy Centre, Madras, for over two years. When it was first introduced, a comparison between this method and the conventional ZiehlNeelsen method was undertaken to test their relative sensitivities, and to see whether fluorescence microscopy yielded false positive results. The results of this comparison are described.

\section{Methods}

Fluorescence microscopy: Equipment.-The light source was a Zeiss multi-purpose microscope lamp III and lamp holder containing an Osram maximum pressure mercury vapour lamp, HBO74, operating from a mains connecting device. A heat-absorbing filter,

* Under the joint auspices of the Indian Council of Medical Research, the Madras Government, the World Health Organization, and the British Medical Research Council. 
and an exciter filter, BG12, were fitted to the microscope lamp. A mounted eyepiece barrier filter, OG5, was attached to the draw tube of the Olympus monocular microscope. The microscope was equipped with a Watson $16 \mathrm{~mm}$. $\times 10$ semi-apochromatic objective, a $4 \mathrm{~mm}$. $\times 40$ parachromatic objective for "uncovered specimens", a $\times 10$ compensating eyepiece and a diamond objective marker. The usual parachromatic $16 \mathrm{~mm}$. and $4 \mathrm{~mm}$. objectives supplied as standard equipment for most microscopes could be used with only slightly less satisfactory results.

Staining (Lempert, 1944)

Auramine phenol.-Dissolve phenol crystals, $30 \mathrm{~g}$. in one litre of distilled water. Warm to $40^{\circ} \mathrm{C}$. and add auramine $0.3 \mathrm{~g}$., shaking vigorously. Filter and store in a dark bottle.

Acid-alcohol,-Dissolve sodium chloride, $20 \mathrm{~g}$. and concentrated hydrochloric acid, 20 c.c. in 500 c.c. distilled water. Add 74 O.P. alcohol, 1,500 c.c.

Potassium permanganate. $-0.1 \%(\mathrm{w} / \mathrm{v})$ in water.

Smears were heat fixed, stained for 6 minutes with auraminephenol without heating, washed, decolourized with acid-alcohol for 2 minutes, washed and counter-stained with potassium permanganate for 30 seconds.

Ziehl-Neelsen microscopy.-The Ziehl-Neelsen procedure was a standard one (Mackie and McCartney, 1956), in which malachite green was used as a counter-stain. Smears were examined with a $\times 7$ eyepiece and a $2 \mathrm{~mm} . \times 100$ oil-immersion objective.

Culture.-Sputum was treated for 15 minutes with about 4 times its volume of $4 \%$ sodium hydroxide. After centrifuging for 15 minutes, the supernatant was discarded. Distilled water was added to the deposit and after further centrifuging two $5 \mathrm{~mm}$. loopfuls of the deposit were added, one to each of two slopes of Lowenstein-Jensen medium. The cultures were incubated for 8 to 9 weeks before being considered negative.

Sputum specimens.-A consecutive series of 1,383 sputum specimens that were cultured were examined by both smear methods. Of these, $981(70.9 \%)$ were from patients who were not receiving chemotherapy. The remaining $402(29.1 \%)$ were from patients during their first 6 months of chemotherapy, almost all with isoniazid and PAS.

Comparison of Huorescence and Ziehl-Neelsen microscopy.Duplicate smears were made from each sputum specimen before treatment with sodium hydroxide. One was examined by fluorescence microscopy and the other after Ziehl-Neelsen staining by different technicians reading independently of each other. The result obtained by one method was recorded without knowledge of the result by the other method. The technicians who carried out the examinations had 
some experience of Ziehl-Neelsen stains, but little of fluorescence microscopy. The results analysed are those obtained before checking by a senior member of the laboratory staff, and are, therefore, typical of the standards that reasonably competent technicians can obtain.

Positive smears were graded into three degrees of positivity, "scanty", "moderate", and "heavy". No particular attempt was made to ensure that these categories were identical for the two smear methods. A smear was called positive when it contained a minimum of 3 or 4 acid-fast bacilli of typical morphology.

\section{Results}

The smear and culture results on the 1,383 sputum specimens are given in the Table. Positive cultures were obtained from $655(47.4 \%)$ of the specimens, and among these, $405(29.3 \%$ of the total specimens $)$ were positive by both smear methods, $36(2.6 \%)$ by fluorescence microscopy only and $28(2.0 \%)$ by Ziehl-Neelsen microscopy only. The remaining 186 culture-positive specimens were negative by both methods. Thus, fluorescence microscopy yielded a slightly larger number of positive smear results which were confirmed by culture, but the difference is not statistically significant.

TABLE

Smear and culture results on 1,383 sputum specimens

\begin{tabular}{|c|c|c|c|c|}
\hline \multirow{2}{*}{$\begin{array}{l}\text { Culture } \\
\text { result. }\end{array}$} & \multicolumn{2}{|c|}{ SMEAR RESULT: } & \multicolumn{2}{|c|}{ SPECIMENS: } \\
\hline & $\begin{array}{l}\text { Fluorescence } \\
\text { microscopy. }\end{array}$ & $\begin{array}{c}\text { Ziehl- } \\
\text { Neelsen } \\
\text { microscopy. }\end{array}$ & Number. & Percentage. \\
\hline Positive & $\left\{\begin{array}{l}\text { Pos } \\
\text { Pos. } \\
\text { Neg. } \\
\text { Neg. }\end{array}\right.$ & $\begin{array}{l}\text { Pos. } \\
\text { Neg. } \\
\text { Pos. } \\
\text { Neg. }\end{array}$ & $\begin{array}{r}405 \\
36 \\
28 \\
186\end{array}$ & $\begin{array}{r}29.3 \\
2.6 \\
2.0 \\
13.4\end{array}$ \\
\hline Negative & $\left\{\begin{array}{l}\text { Pos } \\
\text { Pos. } \\
\text { Neg. } \\
\text { Neg. }\end{array}\right.$ & $\begin{array}{l}\text { Pos. } \\
\text { Neg. } \\
\text { Pos. } \\
\text { Neg. }\end{array}$ & $\begin{array}{r}11 \\
4 \\
3 \\
681\end{array}$ & $\begin{array}{r}0.8 \\
0.3 \\
0.2 \\
49.3\end{array}$ \\
\hline Contaminated & $\ldots$ & $\ldots$ & 29 & 2.1 \\
\hline Total & $\ldots$ & $\ldots$ & 1,383 & 100.0 \\
\hline
\end{tabular}

Among the 699 culture-negative specimens positive smears were found in $18(1.3 \%$ of all specimens). Of these, the smears were positive by both methods in $11(0.8 \%)$ instances, leaving $4(0.3 \%)$ 
which were positive by fluorescence microscopy only and $3(0.2 \%)$ by Ziehl-Neelsen microscopy only. These results show that fluorescence microscopy did not yield smear-positive, culture-negative specimens (which might indicate false positive results) more frequently than did Ziehl-Neelsen microscopy.

From these figures, it will be seen that discrepant results with the two methods were more frequent in positive smears from culturenegative specimens (39\% of 18 specimens) than from culture-positive specimens (14\% of 469 specimens). This finding suggests that the excess with culture-negative specimens may be due to false positive smear results with one or both methods. However, the chance of a discrepant result was greater with a scanty positive than with a more heavily positive smear, and scanty positive smears were very much commoner in culture-negative than in culture-positive specimens. The effects of this association may be taken into account, and thus the possibility that there were false positive smear results with culture-negative specimens may be studied more precisely, in the following manner: Considering Ziehl-Neelsen microscopy first, there were 225 scanty positive smears in culture-positive specimens, and of these $27(12.0 \%)$ were smear-positive by this method alone. Applying the same proportion to the 14 scanty positive smears in culture-negative specimens (all 14 of the Ziehl-Neelsen positive smears were scanty positive), the expected number of smears positive by Ziehl-Neelsen microscopy alone would have been 1.7 (i.e. 12.0\% of 14); the number actually found was 3. A calculation on similar lines shows that the expected number of specimens yielding smears positive by fluorescence microscopy alone would be 2.4 , whereas 4 were found. The difference between the numbers found and expected are small, and similar for both smear methods. Thus, there is again no evidence that false smear-positive results were being found among the culture-negative specimens by fluorescence microscopy.

Among sputa which yielded positive cultures, the percentages yielding negative, scanty, moderate and heavy smear gradings were $33.9,34.4,21.5$ and 10.2 by Ziehl-Neelsen microscopy, and 32.7, $25.5,29.5$ and 12.4 by fluorescence microscopy. These data show a tendency for fluorescence microscopy to yield slightly higher gradings. Considering these specimens which were positive by only one of the smear methods, moderate gradings were assigned to 3 of the 36 smears positive by fluorescence microscopy only; and to 1 of the 28 smears positive by Ziehl-Neelsen microscopy only; the remainder were graded as scanty. Thus, taking into account the overall results of the grading of all culture-positive specimens, there was a similar distribution of smear positivity among those positive by only one smear method. These results from specimens proved positive by culture suggest that fluorescence microscopy is no more likely to yield false negative results than is Ziehl-Neelsen microscopy. 


\section{Discussion}

Our comparison has shown that fluorescence microscopy reveals positive smears as often as does Ziehl-Neelsen microscopy. Moreover, there is no evidence that it yields any appreciable number of false positive or false negative results. The main advantage of fluorescence microscopy is a very great saving of time in the preparation and examination of smears. The area of the slide included in one field is about 50 times larger than with Ziehl-Neelsen microscopy, so that fewer fields need to be examined. Furthermore, the staining procedure is simpler, since no heating is required, and there is no need to use immersion oil during microscopy. In practice, the preparation, staining and examination of 100 smears is less than a day's work for one technician and their examination alone can easily be done in 2 hours. In comparison, the preparation and examination of the same number of smears takes at least twice as long if they are stained by the Ziehl-Neelsen method and examined with an oil-immersion lens. A second advantage is the saving in initial cost of equipment. A busy laboratory might require two microscopes for Ziehl-Neelsen microscopy but could examine the same number of smears by fluorescence microscopy with one. The cost of the additional equipment for the latter method is less than the cost of an additional microscope. The cost of replacing the mercury vapour lamps is much less than the salary of a technician.

Although fluorescence microscopy has the advantage of speed and cheapness, it requires more skill for its operation. The optical equipment needs careful adjustment to get maximum light transmission and it is, therefore, advisable to clamp the lamp holder and microscope permanently in position on the bench. If the electrical supply is interrupted the lamp cannot be relit for at least 3 hours after. Thus, it is necessary to have a spare lamp and to be able to change it easily. Skill is also required to distinguish with certainty acid-fast bacilli from other small naturally fluorescent particles present in some smears. When first using fluorescence microscopy, it is necessary to examine all small fluorescent objects seen both with the $\times 10$ and $\times 40$ objectives. With practice it becomes possible to distinguish bacilli with a fair degree of certainty under the $\times 10$ objective only, so that almost all negative smears can be examined with this objective only. However, it is always necessary to confirm the bacillary morphology with the higher power when the smears are scantily positive. Finally, if any doubt remains, it is possible to ring individual suspicious objects with the diamond objective marker, then re-stain, over the fluorescence stain, by the Ziehl-Neelsen method, and examine with an oil-immersion lens. When smears are being examined by technicians, it is wise for a more senior member of the staff to check those that are judged to be positive. This only takes a few minutes each day, whereas checking of Ziehl-Neelsen 
positives would take much longer.

Fluorescence microscopy can be recommended for the larger laboratory that examines at least 40 direct smears a day and where some supervision of the work of technicians is possible. Under these circumstances, it saves time and the initial cost of equipment. For the smaller laboratory it cannot be recommended so freely, since the need for greater skill in its use might lead to poor results.

\section{Summary}

The equipment and method for the examination of smears for tubercle bacilli by fluorescence microscopy is described. A comparison with the conventional Ziehl-Neelsen method on 1,383 routine sputum specimens which were also cultured showed that fluorescence microscopy yielded as many positive smears and had no tendency to produce false positive or false negative results. The method can be recommended for the larger laboratory as economical in time and initial expense.

\section{References}

ClifgG, J. W., and Foster-Carter, A. F. (1946) Brit. Jour. Tuherc., 40, p. 98. Von Haebler, T., and Murray, J. F. (1954) $S$. Afr. Med. Joli., 28, p. 45.

Hagemann, P. (1937) Disch. Med. Wschr., 63, p. 514.

Holm, J., and Plum, N. (1943) Acta. Tuberc. Scand., 17, p. 13.

Kuster, H. (1939) Disch. Med. Wschr., 65, p. 92.

LEMPERT, H. (1944) Lancet, 247, p. 818.

Lind, H. E., and Shaughnessy, H. J. (1941) Jour. Lab. Clin. Med., 27, p. 531.

MaCkie, T. J., and MCCARTney (1956) "Handbook of Practical Bacteriology", London, p. 95.

Needham, G. M. (1957) Proc. Mayo Clin., 32, p. 1.

Norman, W. A., and Jelks, F. W. (1945) Bull. Inst. Med. Lab. Tech., 11, p. 37.

Ritterhoff, R. J., and Bowman, M. G. (1945) Amer. Jour. Clin. Path., 15, p. 39. (Techn. Sect.)

Tanner, F. H. (1941) Proc. Mayo Clin., 16, p. 830.

Tanner, F. H. (1948) Amer. Jour. Med. Technol., 14, p. 83.

Wilson, H. M. (1952) Amer. Rev. Tuberc., 65, p. 709. 